TITLE:

\title{
The critical length of shear bands in metallic glass
}

$\operatorname{AUTHOR}(S)$ :

Matsumoto, R.; Miyazaki, N.

\section{CITATION:}

Matsumoto, R. ... [et al]. The critical length of shear bands in metallic glass. Scripta Materialia 2008, 59(1): 107-110

ISSUE DATE:

2008-01

URL:

http://hdl.handle.net/2433/88964

\section{RIGHT:}

Copyright (c) 2008 Acta Materialia Inc.; This is not the published version. Please cite only the published version.; この論文は出版社版でありませ ん。引用の際には出版社版をご確認ご利用ください。 


\title{
Critical Length of Shear Band in Metallic Glass
}

\author{
R. Matsumoto ${ }^{\mathrm{a}}$ and N. Miyazaki ${ }^{\mathrm{a}}$ \\ ${ }^{a}$ Department of Mechanical Engineering and Science, Graduate School of Engineering, Kyoto University, \\ Yoshida-Honmachi, Sakyo-ku, Kyoto, 606-8501, Japan
}

\begin{abstract}
In order to exploit the potential applications of bulk metallic glasses (BMGs), it is essential to improve their ductility. In this study, we first performed a molecular dynamics (MD) simulation of the propagation of a shear band (SB) through a BMG plate, and analyzed the evolution of temperature and shear stress distributions in the plane of the SB. Using the MD simulation results and fracture mechanics, we then obtained the critical length of SBs, which potentially cause catastrophic propagation.
\end{abstract}

Keywords: Bulk metallic glasses; Shear Bands; Ductility; Fracture; Molecular Dynamics

Unlike most conventional crystalline metals and alloys, BMGs have many excellent properties [1-3], such as great hardness, a high elastic limit, and good resistance to fatigue and corrosion. The deformation and fracture properties of BMGs at room temperature are controlled by the initiation and propagation of SBs. In fact, at room temperature, BMGs usually show limited global deformation of about $2 \%$, particularly under tensile deformations, due to the catastrophic propagations of SBs. On the other hand, the literature also reports that BMGs with a particular material composition have greater plasticity under compressive deformations due to the nucleation of multiple micro SBs $[4,5]$. In order to achieve a wider range of possible applications of BMGs, it is therefore crucial to clearly elucidate their shear banding behavior.

SBs in monolithic BMGs are roughly planar and nucleate preferentially from the surface, with intense heating and evidence of melting at the trailing edges [6,7]; the width of such SBs was reported as $w=10 \sim 20 \mathrm{~nm}$ 
[7 9]. Recently, F. Shimizu et al. studied the critical condition for yielding BMGs, and proposed that it corresponds to embryonic shear band (ESB) propagation [10]. They proposed that, for an ESB to propagate, far-field shear stress must exceed the quasi-steady-state glue traction $\tau_{\text {glue }}$ of shear-alienated glass until the glass transition temperature is reached internally due to frictional heating. At this point, the ESB matures as a runaway shear crack. The incubation length scale necessary for this maturation is estimated to be $0.1 \sim 1 \mu \mathrm{m}$ for $\mathrm{Zr}$-based BMGs. However, even if melting occurred inside a SB and it turned into a crack, the length of the SB (or crack) must exceed a critical length to cause spontaneous growth, because according to fracture mechanics, energy release rate of crack controls the fracture, and not the initiation of the crack. In this study, we performed a molecular dynamics (MD) simulation of mode II deformation of a notched BMG plate. From this, we discuss the results in the light of fracture mechanics by assuming a SB in a BMG as a crack that includes a viscous fluid, and we estimate the critical SB length in a BMG under tension and compression.

The shifted Lennard-Jones (L-J) potential [11], whose parameters were defined based on Inoue's three basic principles [2] to generate BMGs, was used in this study. A thin square plate with an amorphous structure, here we call BMG plate, was numerically produced by melting-rapid quenching MD simulations [11] subject to three dimensional periodic boundary conditions. The system contained elements $a, b$, and $c$ [11] in 1:8:1 ratio was used.

In this analysis, a notched BMG plate was used, and a primary SB was forced to nucleate and propagate from the notch bottom (see Figure 1). By arranging three BMG plates in the $\mathrm{x}$ direction and two in the $\mathrm{y}$ direction, we obtained a large BMG plate of dimensions $L_{x} \times L_{y} \times L_{z}=360 \times 244 \times 1.8 \mathrm{~nm}$, composed of about 5.6 million atoms. The periodic boundary conditions in the $\mathrm{x}$ and y directions were removed, and a notch with length $L_{\mathrm{c}}=50 \mathrm{~nm}$ and bottom radius $R_{\mathrm{c}}=1 \mathrm{~nm}$ was introduced at the center of the left side of the BMG plate. The first step was then to allow relaxation for $60 \mathrm{ps}$ under a constant temperature of $1 \mathrm{~K}$. During the relaxation, the boundary atoms, i.e., those located within the cut-off distance of the interatomic potential $\left(r_{\mathrm{c}}=0.768 \mathrm{~nm}\right)$ from the left, top, and bottom edges of the plate, are fixed along the $\mathrm{x}$ and $\mathrm{y}$ directions. After the relaxation, the boundary atoms in the upper and lower half of the left boundary are moved toward the right and left, respectively. During the first 2 ps of deformation, the shear displacement rate is accelerated linearly until a speed of $v_{\mathrm{s}}=200 \mathrm{~m} / \mathrm{s}$ is reached in order to 
suppress the propagation of elastic waves.

Figure 2(a) shows the temperature distribution at $560 \mathrm{ps.} \mathrm{A} \mathrm{band-like} \mathrm{high} \mathrm{temperature} \mathrm{region} \mathrm{appears}$ ahead of the notch bottom. Figure 2(b) shows the evolution of temperature distribution along the plane of the SB. The temperature was estimated from a region of width $2 \mathrm{~nm}$, and an intense temperature rise occurred at the notch bottom. However, this temperature rise decreased after $t>400 \mathrm{ps}$, and then the high temperature region began to grow further. Figure 2(c) shows the time evolution of the maximum temperature $T_{\max }$ ahead of the notch bottom, obtained by the means of the MD simulation and the following theoretical estimation [12, 13]. By treating a SB as a heat source of zero thickness in an infinite medium, the temperature rise $\Delta T$ at the center of the SB is expressed as ,

$$
\Delta T=\frac{\tau_{\mathrm{f}} v_{\mathrm{p}}}{\sqrt{\pi \rho C K}} \times \sqrt{t},
$$

where $\tau_{\mathrm{f}}$ is the flow stress, $v_{\mathrm{p}}$ is the plastic displacement rate in the plane of the $\mathrm{SB}, \rho$ is the density, $C$ is the specific heat, $K$ is the thermal conductivity, and $t$ is the time elapsed from the beginning of shear banding. For this L-J system, $\tau_{\mathrm{f}}=0.9 \mathrm{GPa}, \rho=3,700 \mathrm{~kg} / \mathrm{m}^{3}, C=462 \mathrm{~J} /(\mathrm{kg} \cdot \mathrm{K})$, and $K=0.87 \mathrm{~W} /(\mathrm{m} \cdot \mathrm{K})$. The plastic displacement rate is $v_{\mathrm{p}} \sim v_{\mathrm{s}}=200 \mathrm{~m} / \mathrm{s}$. Although the evolution of the maximum temperature obtained by the MD simulation is proportional to $t^{1 / 2}$ until $t \sim 400 \mathrm{ps}$, the actual value of the temperature is almost half of the theoretical prediction. This is due to the two-dimensional effect of the thermal diffusion as heat spreads along the SB, and due to the finite width of the SB. As stated above, the temperature rise becomes moderate compared to the curve proportional to $t^{1 / 2}$ after $t \sim 400 \mathrm{ps}$. The temperature at this point is about $0.75 T_{\mathrm{g}}$, where $T_{\mathrm{g}}$ is the glass transition temperature $(\sim 1,400 \mathrm{~K})$. Figure 2(d) shows the evolution of the shear stress distribution along the SB. The shear stress was estimated from the region where the temperature distribution was estimated in Fig. 2(b), i.e. 2 nm width region ahead of the notch bottom. As the temperature rises, the shear stress decreases. However, the shear stress remains at $0.7 \mathrm{GPa}$ throughout the entire simulation.

The displacement-controlled boundary conditions are used in this MD analysis. We see that if the shear displacement velocity $v_{\mathrm{s}}$ is increased, the shear stress is also increased, and therefore, the temperature inside the SB can rise faster and reach a much higher temperature. It is considered that the temperature rises until the heat 
generation in the SB and the heat diffusion from the SB are balanced. We define the temperature at which the temperature change becomes negligibly small as $T_{\mathrm{f}}$, and the shear stress at this condition as traction stress $\tau_{\mathrm{t}}$.

BMGs show Newtonian behavior in the high temperature range, and the viscosity $\eta$ is about $0.01 \mathrm{~Pa} \cdot \mathrm{s}$ near the melting point $[14,15]$. If we used a SB width $w=20 \mathrm{~nm}[7-9]$ and shear displacement velocity $0.5 v_{\mathrm{R}}$, where $v_{\mathrm{R}}$ is the velocity of a Rayleigh wave $\sim 2,000 \mathrm{~m} / \mathrm{s}$ for Zr-based BMGs, the traction stress $\tau_{\mathrm{t}}$ is estimated to be $\sim 0.5 \mathrm{GPa}$, and this value is the order of the external stress. In fact, the L-J system used here also yields very high shear stress $(\sim 0.3 \mathrm{GPa})$ even near the melting point $(2,250 \mathrm{~K})$ under such an extremely high strain rate $\left(\mathrm{d} \gamma / \mathrm{d} t=5 \times 10^{10} \mathrm{~s}^{-1}\right)$.

We demonstrated that a high temperature region, perhaps even a liquid or a near-liquid region, definitely appears inside a running SB, and that this region grows further. Unfortunately, we cannot capture the entire shear banding process within the available time- and spatial-scale of the MD analysis. However, based on the MD results, the temperature and shear stress distributions in a running SB can be modeled, as shown in Fig. 3(a). The shear stress far from the SB is small compared to the shear stress near the tip, and if the SB is sharp enough, the stress singularity corresponding to the crack appears. A plastic region exists at the leading part of the SB, where intense heating occurs, and shear stress decreases due to temperature rise. In the following part of the SB, the changes in the temperature and shear stress are considered enough small. For convenience, we call this region as the liquid region. For simplicity, we assume that the traction stress $\tau_{\mathrm{t}}$, the flow stress in the plastic region $\tau_{\mathrm{f}}$, and the shear displacement velocity along the SB $v_{\mathrm{s}}$ are all constant, and we equivalence the SB as a Mode II crack that includes a viscous liquid, as shown in Fig. 3(b). In the present modeling, we also take into account the propagation velocity of SB $v_{\mathrm{g}}$, because only propagating SB can generate liquid region inside the SB.

Spontaneous crack growth occurs when the energy release rate of a crack $G$ exceeds the energy required to extend the crack per unit length. Here, we assume that the length of the plastic region ahead of the liquid region $a_{\mathrm{p}}$ is constant during the extension of the SB. Under this assumption, since the amount of growth of the SB (or shear crack) agrees with the extension length of the liquid region, the condition for the unstable propagation of the SB is given by $G=\gamma_{\mathrm{m}}$, where $\gamma_{\mathrm{m}}$ is the energy required to melt a unit length of the shear banding region.

The energy release rate of a Mode II crack, which includes a viscous liquid, with finite growth velocity 
$v_{\mathrm{g}}$ is expressed as follows $[16,17]$ :

$$
G \approx\left(1-\frac{v_{g}}{v_{R}}\right) \frac{K_{\mathrm{II} \_ \text {eff }^{2}}^{E^{\prime}} .}{\text {. }}
$$

Here, $E^{\prime}$ is equal to $E=\left(1-v^{2}\right)$, which is the under plane-strain, and $E$ is under plane-stress condition, where $E$ and $v$ are Young's modulus and Poisson's ratio, respectively. $K_{\text {II_eff }}$ is the stress intensity factor, which considers the traction stress acting on the crack surface, and is expressed as $K_{\mathrm{II} \_ \text {eff }}=K_{\mathrm{II}}-K_{\mathrm{t}}$, where $K_{\mathrm{II}}=\tau^{\infty} \sqrt{\pi a_{\mathrm{L}}}$, and $K_{\mathrm{t}}=\tau_{\mathrm{t}} \sqrt{\tau a_{\mathrm{L}}}=\left(\eta v_{\mathrm{s}} / w\right) \sqrt{\pi a_{\mathrm{L}}}$ under constant traction stress. The energy release rate $G$ decreases as both $v_{\mathrm{s}}$ and $v_{\mathrm{g}}$ increase.

On the other hand, $\gamma_{\mathrm{m}}$ is expressed as follows:

$$
\gamma_{\mathrm{m}}=\int_{0}^{t_{\mathrm{f}}} \tau_{\mathrm{f}}(\mathrm{t}) \nu_{\mathrm{s}}(t) \mathrm{d} t=\frac{\pi \rho C K\left(T_{\mathrm{f}}-T_{\mathrm{r}}\right)^{2}}{\tau_{\mathrm{f}} v_{\mathrm{s}}}
$$

Here, $t_{\mathrm{f}}$ is the time taken for the internal temperature to reach sufficiently close to $T_{\mathrm{f}}$, and it was estimated using Eq. (1). Since the temperature rise inside a SB is lower than that estimated by Eq. (1) (as shown in Fig. 2(c)), Eq. (3) gives a smaller $\gamma_{\mathrm{m}}$, i.e., a safe estimate of the critical SB length. The resistance $\gamma_{\mathrm{m}}$ decreases as $v_{\mathrm{s}}$ increases, because energy loss caused by the thermal diffusion decreases.

From Eq. (2) and (3), the critical length of the liquid region $a_{\mathrm{L}_{-} \mathrm{c}}$ is calculated as follows:

$$
\begin{aligned}
& a_{\mathrm{L}_{-} \mathrm{c}}=D \frac{1}{\alpha_{\mathrm{s}}\left(1-\alpha_{\mathrm{g}}\right)\left(1-V \alpha_{\mathrm{s}}\right)^{2}} \\
& D=\frac{\rho C E^{\prime} K\left(T_{\mathrm{f}}-T_{\mathrm{r}}\right)^{2}}{\tau_{\mathrm{f}} \tau^{\infty^{2}} v_{\mathrm{R}}}, \quad V=\frac{\eta v_{\mathrm{R}}}{w \tau^{\infty}} .
\end{aligned}
$$

Here, $\alpha_{\mathrm{s}}=v_{\mathrm{s}} / v_{\mathrm{R}}$ and $\alpha_{\mathrm{g}}=v_{\mathrm{g}} / v_{\mathrm{R}}$, and $D$ is a parameter with a length dimension that has a strong influence on the critical SB length, i.e., ductility. $V$ is the ratio between the external stress $\tau^{\infty}$ and the traction stress $\tau_{\mathrm{t}}$ at $v_{\mathrm{S}}=v_{\mathrm{R}}$. When the length of the liquid region exceeds $a_{\mathrm{L}_{-} \mathrm{c}}$ and the SB begins to unstable growth, it is impossible to arrest this growth without a large stress gradient or inhomogeneity in the material's properties, and this structure would cause a catastrophic shear-banding fracture.

Because BMGs also generally include initial inhomogeneities such as stress concentration zones and 
residual stresses, the external stress $\tau^{\infty}$ is smaller than the flow stress $\tau_{\mathrm{f}}$. Moreover, we can reasonably assume that $\tau_{\mathrm{t}}<\tau^{\infty}$ and $0<\alpha_{\mathrm{s}} \leq \alpha_{\mathrm{g}}<1$. Under these conditions, $a_{\mathrm{L}_{-} \mathrm{c}}$ has one minimum value $a_{\mathrm{L}_{-} \text {min }}$ with respect to $\alpha_{\mathrm{s}}\left(=\alpha_{\mathrm{g}}\right)$, and a SB with a longer liquid region than $a_{\mathrm{L}_{-} \min }$ could potentially cause catastrophic fracture. In order to retain the liquid region, the heat generation at the center of the SB due to viscous flow must be greater than the outward flow of heat. Therefore, the internal temperature $T_{\mathrm{f}}$, the width $w$ of the SB, and the shear velocity $v_{\mathrm{s}}$ are correlated in a non-trivial manner. Since the viscosity of BMGs is about $0.01 \mathrm{~Pa} \cdot \mathrm{s}$ at a temperature of around $2 T_{\mathrm{g}}$, and the decrease rate of the viscosity becomes smaller at a higher temperature [14], we assume that $T_{\mathrm{f}}=2 T_{\mathrm{g}}$ and $\eta=0.01 \mathrm{~Pa}$ - s. We also use $w=20 \mathrm{~nm}, \tau_{\mathrm{f}} / \tau^{\infty}=1.25$, and $T_{\mathrm{r}}=300 \mathrm{~K}$. Using the typical physical values of a Zr-based BMG [2, 18, 19]: $E=83.4 \mathrm{GPa}, v=0.37, \rho=6,720 \mathrm{Kg} / \mathrm{m}^{3}, K=10 \mathrm{~W} /(\mathrm{J} \cdot \mathrm{K}), C=500 \mathrm{~J} /(\mathrm{kg} \cdot \mathrm{K}), T_{\mathrm{g}}=657 \mathrm{~K}, \tau^{\infty}=0.89 \mathrm{GPa}$, and $v_{\mathrm{R}}=2,000 \mathrm{~m} / \mathrm{s}$, we obtain $a_{\mathrm{L} \_\min }=45 \mu \mathrm{m}$ when $v_{\mathrm{s}}=v_{\mathrm{g}}=389 \mathrm{~m} / \mathrm{s}$. The heat generation in the plastic region $\left(\gamma_{\mathrm{m}}\right)$ is estimated to be $316 \mathrm{~J} / \mathrm{m}^{2}$, and this result agrees well with the lower bound estimate of $400 \mathrm{~J} / \mathrm{m}^{2}$, reported by J. J. Lewandowski et al. [6]. The BMG-samples with $\mu \mathrm{m}$ size show large compressive plastic deformations [20], and each shear displacement estimated from the serration observed in the stress-strain curves obtained from the compression, generated by one continuous slip, is shorter than $\sim 8 \mu \mathrm{m}[12,21]$. These facts indicate that only SBs with length $>a_{\mathrm{L} \_ \text {min }}$ propagate catastrophically.

Since delamination can occur at the trailing edge under tensile conditions, we neglect viscosity $(V=0)$. In this case, the temperature need not be raised until the viscosity $\eta$ is sufficiently decreased, i.e., until the traction stress $\tau_{\mathrm{t}}$ decreases. Therefore, if $T_{\mathrm{f}}=T_{\mathrm{g}}$ is assumed, we can obtain $a_{\mathrm{L}_{-} \min }=1.8 \mu \mathrm{m}$ when $v_{\mathrm{s}}=v_{\mathrm{g}}=0.5 v_{\mathrm{R}}$. This length is approximately equal to the incubation length scale necessary for melting, which was estimated by F. Shimizu et al. [10]. Therefore, the initiation of the crack leads directly to unstable propagation when $V=0$. The differences in the yield stress and the shear angle between compression and tension tests are explained by the Mohr-Coulomb-type yield criterion of micro deformations [21-23]. We believe that the difference in $a_{\mathrm{L} \_ \text {min }}$ between the cases with and without the viscosity effects also affects the difference in ductility. Recently, the tensile ductility and necking were also observed using BMG samples with dimensions of the order of $100 \mathrm{~nm}$ [24], and this size is smaller than $a_{\mathrm{L} \_ \text {min }}$. 
The length of the liquid region generated by a continuous slip must be maintained shorter than $a_{\mathrm{L}_{-}}$. When the growth of the SB is stopped and the SB is cooled, although damages related to the free volume may linger [9], the crack disappears. Here, we discuss how we can improve the ductility of BMGs by increasing the critical SB length $a_{\mathrm{L}_{-} c}$. A larger value for the parameter $D$ or $V$ results in a larger value of $a_{\mathrm{L}_{-} \min }$. An effective approach to achieve this is, unfortunately, to decrease $\tau^{\infty}$ by introducing inhomogeneities of the micro structure, because the $\tau^{\infty}$ has a strong influence on $D$. Unlike $\tau^{\infty}$, other parameters cannot be changed drastically, because they are almost completely determined by the material's composition. It is important to note that although an increase in $V$ contributes to an increase in $a_{\mathrm{L}_{-} \min }$, this approach may fail under loading conditions where delamination occurs in the trailing part of the SBs.

To prevent SB growth by inhomogeneities, such as crystalline particles, may also effective to improve the ductility of BMGs. In this approach, the spacing between inhomogeneities should be much less than $a_{\mathrm{L}_{-} \min }$. This is because once the unstable SBs are generated at the coarse spacing regions, the growth of the SBs cannot stop without the use of some mechanism capable of arresting running shear cracks.

In this study, we first analyzed the evolution of the temperature and shear stress distributions in the plane of the SB by means of MD simulation. The critical length of SBs that could potentially cause catastrophic propagation was then estimated. We also discussed the difference in ductility between compressive and tensile tests, and proposed a strategy to improve the ductility of BMGs.

R. M. acknowledges the support provided by the Ministry of Education, Culture, Sports, Science and Technology of Japan, Grant-in-Aid for Scientific Research on Priority Areas, "Materials Science of Bulk Metallic Glasses.”

[1] A. L. Greer, Science 267 (1995) 1947.

[2] A. Inoue, Acta Mater. 48 (2000) 279.

[3] C. A. Schuh, T. C. Hufnagel, and U. Ramamurty, Acta Mater. 55 (2007) 4067.

[4] J. Schroers and W. L. Johnson, Phys. Rev. Lett. 93 (2004) 255506. 
[5] J. Y. Lee, K. H. Han, J. M. Park, K. Chattopadhyay, W. T. Kim, and D. H. Kim, Acta Mater. 55 (2007) 4067.

[6] J. J. Lewandowski and A. L. Greer, Nature Materials 5 (2006) 15.

[7] B. Yang, M. L. Morrison, P. K. Liaw, R. A. Buchanan, G. Wang, C. T. Liu, and M. Denda, Appl. Phys. Lett. 86 (2005) 141904.

[8] E. Pekarskaya, C. P. Kim, and W. L. Johnson, J. Mater. Res. 16 (2001) 2513.

[9] J. Li, Z. L. Wang, and T. C. Hufnagel, Phys. Rev. B 65 (2002) 144201.

[10] F. Shimizu, S. Ogata, and J. Li, Acta Mater. 54 (2006) 4293.

[11] R. Matsumoto and M. Nakagaki, CMES 10 (2005) 187.

[12] W. J. Wright, R. B. Schwarz, and W. D. Nix, Mater. Sci. Eng. A 319-321 (2001) 3809.

[13] J. D. Eshelby and P. L. Pratt, Acta Metall. 4 (1956) 560.

[14] Y. Kawamura and A. Inoue, Appl. Phys. Lett. 77 (2000) 1114.

[15] C. Way, P. Wadhwa, and R. Bush, Acta Meter. 55, (2007) 2977.

[16] T. L. Anderson, Fracture Mechanics, 3rd ed. (CRC, 2005), 183.

[17] J. R. Rice, Fracture: An Advanced Treatise (Academic Press, 1968), vol. 11, p. 214.

[18] M. Yamasaki, S. Kagao, and Y. Kawamura, Scripta Mater. 53 (2005) 63.

[19] W.-H. Wang, J. Appl. Phys. 99 (2005) 093506.

[20] Q. Zheng, S. Cheng, J. H. Strader, E. Ma, and J. Xu, Scripta Mater. 56 (2007) 161.

[21] T. Mukai, T. G. Nieh, Y. Kawamura, A. Inoue, and K. Higashi, Intermetallics 10 (2002) 1071.

[22] C. A. Schuh and A. C. Lund, Nature Materials 2 (2003) 449.

[23] S. Ogata, F. Shimizu, J. Li, M. Wakeda, and Y. Shibutani, Intermetallics 14 (2006) 1033.

[24] H. Guo, P. F. Yan, Y. B. Wang, J. Tan, Z. F. Zhang, M. L. Sui, and E. Ma, Nature Materials 6, (2007) 735. 


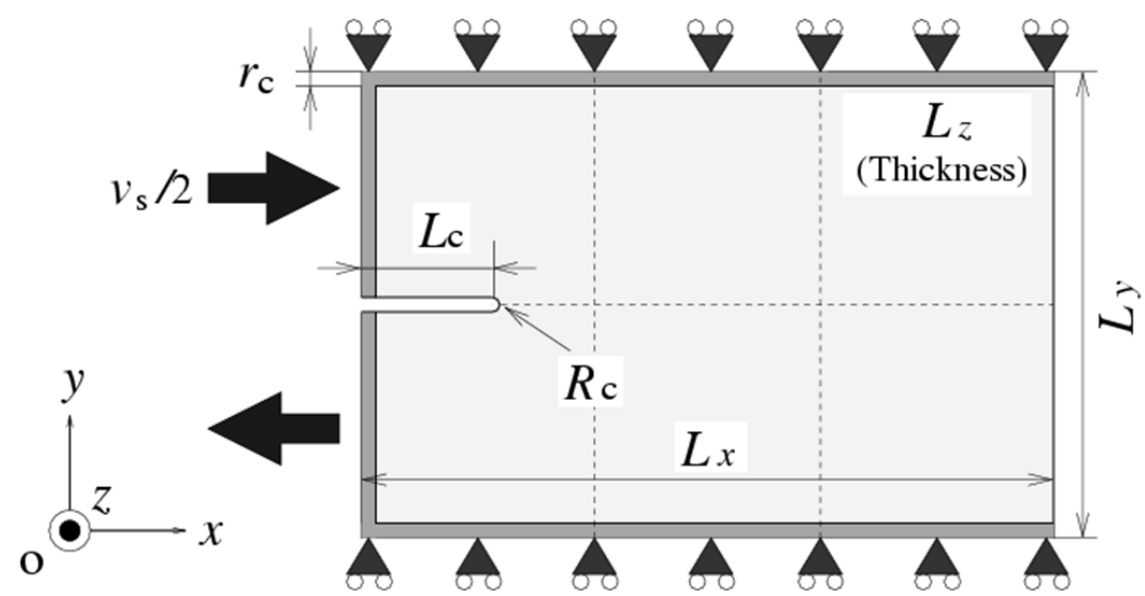

Figure 1. Simulation model for the shear banding analysis: The periodic boundary condition is applied along the $\mathrm{z}$ direction. The motion along the y direction of all the boundary atoms is fixed during the shear banding simulation. 
(a)

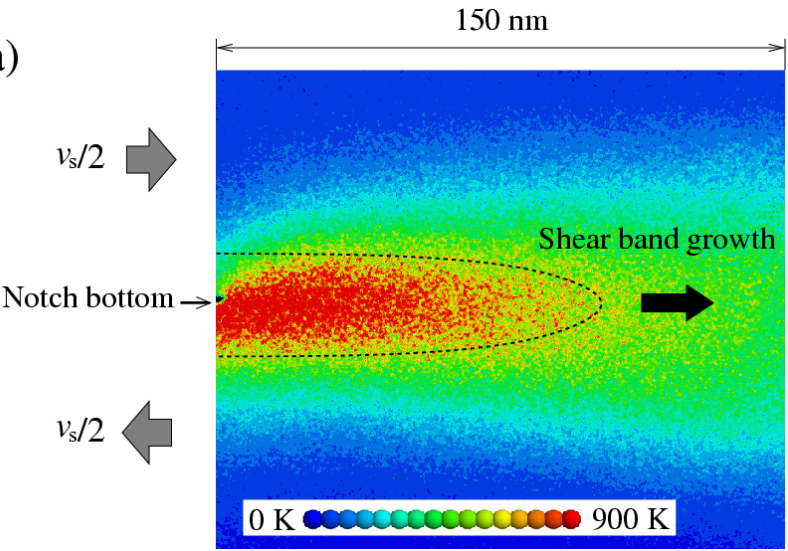

(c)

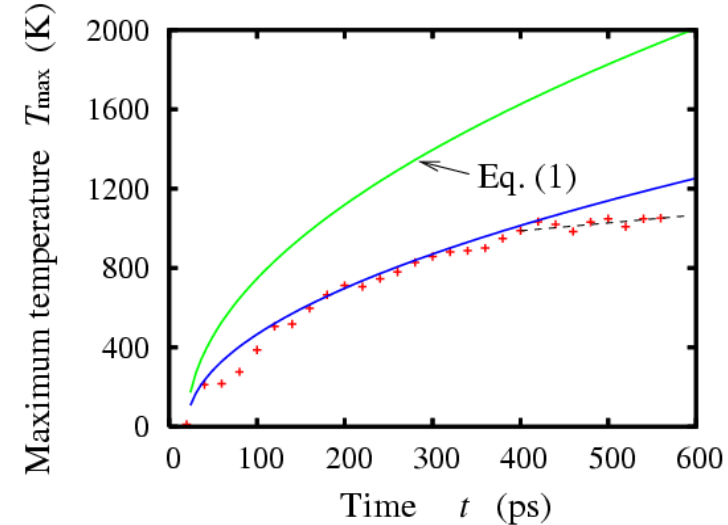

(b)

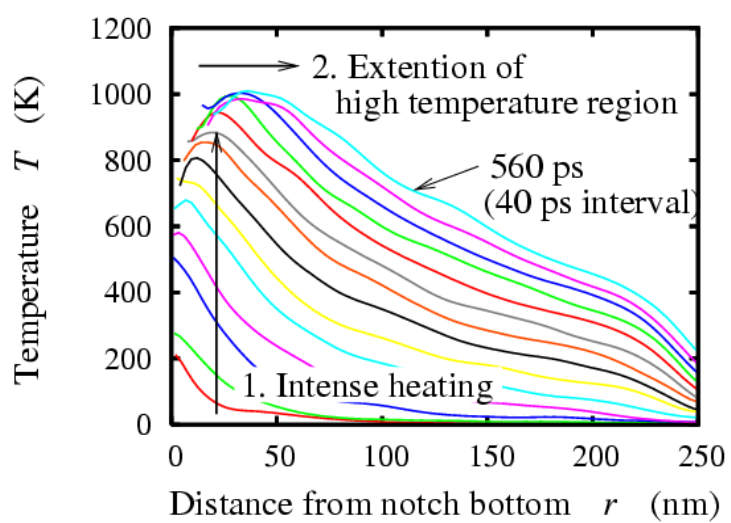

(d)

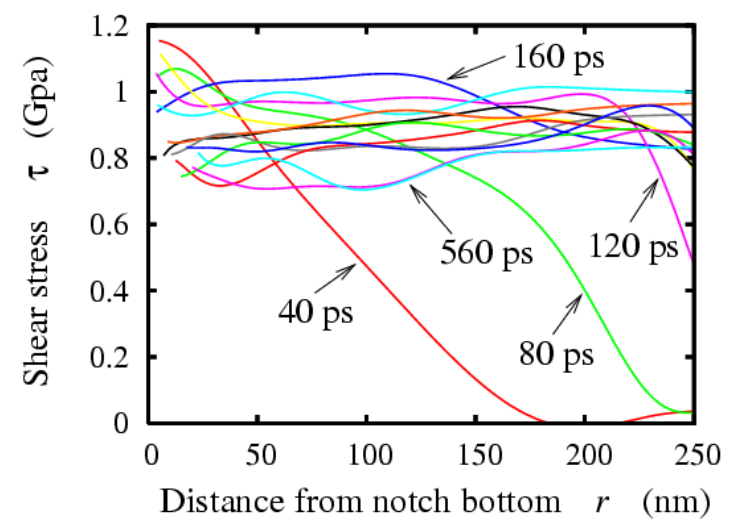

Figure 2. (a) Temperature distribution at $t=560 \mathrm{ps}$. (b) Evolution of temperature distribution ahead of the notch bottom. (c) Evolution of the maximum temperature. (d)Evolution of shear stress distribution. 
(a)

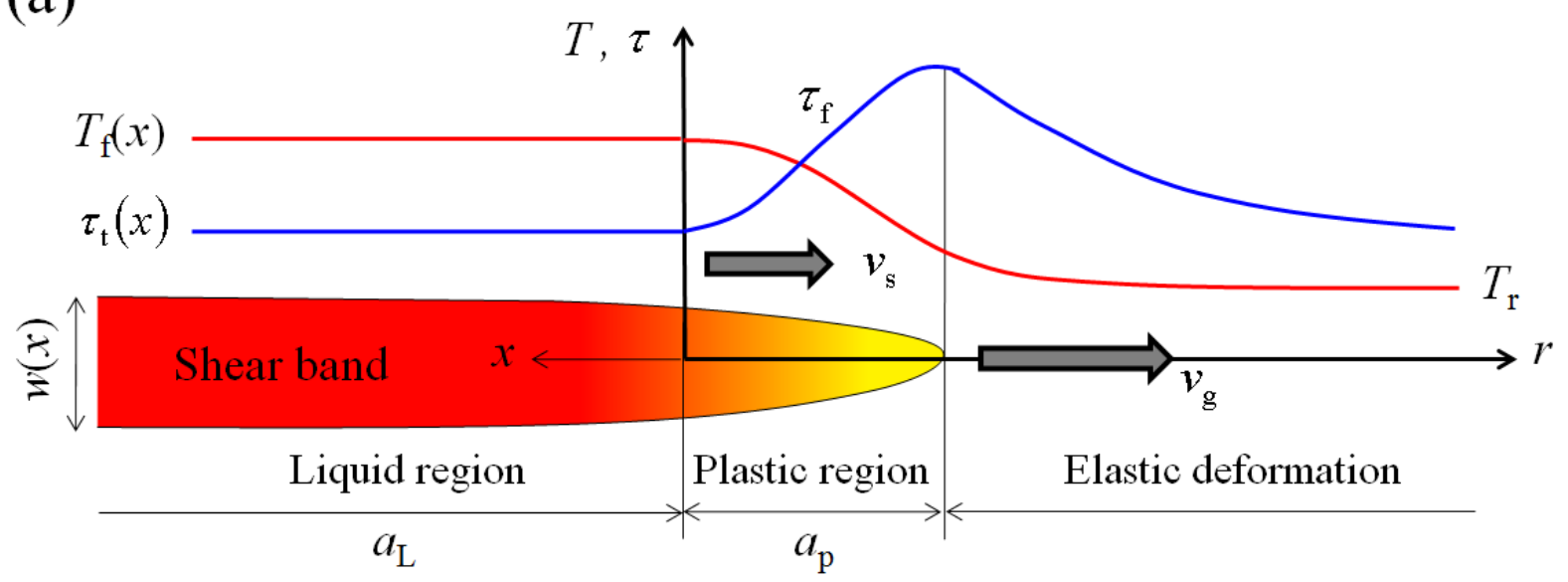

(b)

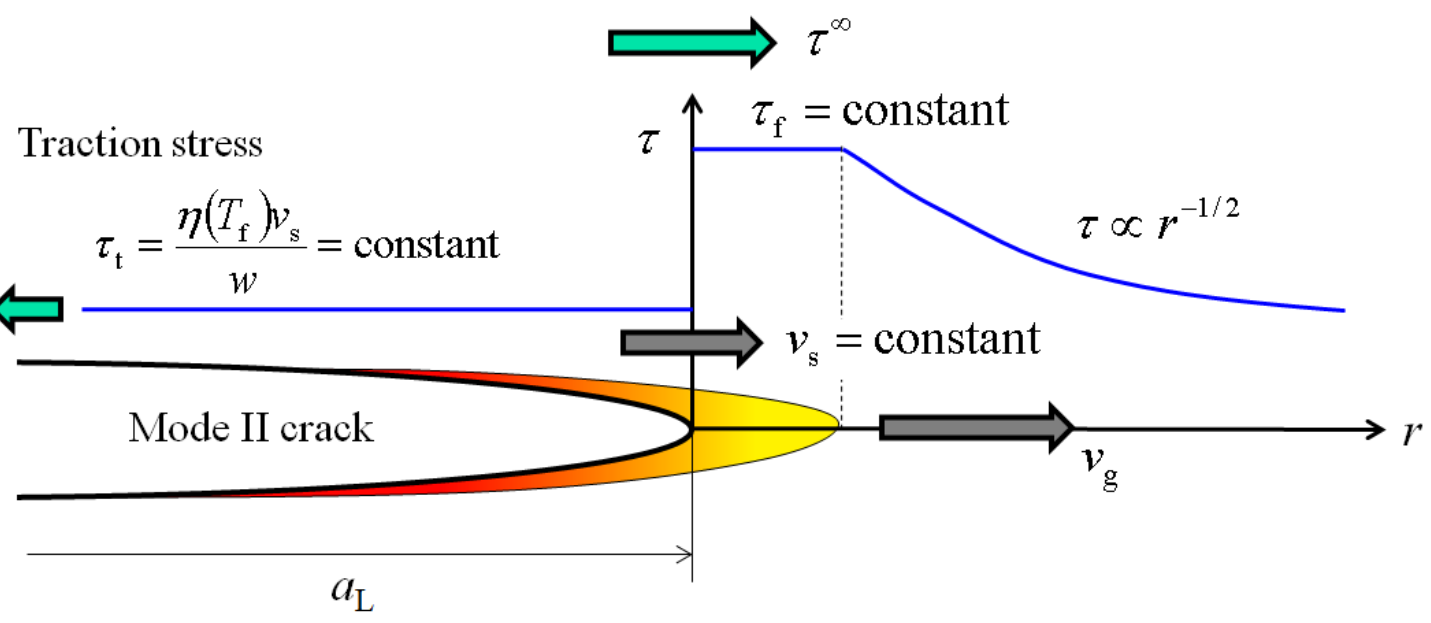

Figure 3. (a) Temperature and shear stress distributions in the shear banding plane: $T_{\mathrm{r}}$ is the room temperature and $\tau_{\mathrm{f}}$ is the flow stress. $T_{\mathrm{f}}$ and $\tau_{\mathrm{f}}$ are the temperature and traction stress in the liquid region, respectively, and $v_{\mathrm{g}}$ and $v_{\mathrm{s}}$ are the growth velocity of the SB and the shear displacement velocity along the SB, respectively. (b) Crack model of SB: $\tau^{\infty}$ is the applied shear stress far from the crack tip. 